\title{
Molecular Characteristics, Recombinant Expression and Activity Detection of OsGSTL1 from Rice
}

\author{
Tingzhang $\mathrm{Hu}^{1,2^{*}}$, Yongwei Yang ${ }^{1}$, Lili Tan ${ }^{1}$, Junnian Yang ${ }^{2}$ and Yingmei $\mathbf{W u}^{2}$ \\ ${ }^{1}$ Key Laboratory of Biorheological Science and Technology; Ministry of Education; College of Bioengineering; \\ Chongqing University; Chongqing - P R - China. ${ }^{2}$ School of Life Science and Engineering; Chongqing Three \\ Gorges University; Chongqing 40400 - P R - China
}

\begin{abstract}
The MRNA of OsGSTL1 was detected in the roots and leaves of rice plants at seedling and tillering stages, and their roots, leaves and panicles at the heading stage. The full-length open reading frame of OsGSTL1 cDNA was $732 \mathrm{bp}$ and encoded a putative polypeptide of 243 amino acids with a calculated molecular mass of $27.30 \mathrm{kDa}$ and a theoretical pI of 5.50. The protein sequences of OsGSTL1 exhibited typical feature of the lambda class GST, which contained the conserved domain "GST_C_Lambda" in C-terminal alpha helical domain and a highly conserved Cys42 in active center. In silico predictions showed that the OsGSTL1 protein was strongly hydrophilic. The phylogenetic analysis revealed OsGSTL1 belonged to monocots subgroup and was closer to IN2-1 of Z. may. The OsGSTL1 gene was cloned into pYTV vector and was introduced into yeast strain PEP4. Western blot analysis showed that the exogenous OsGSTL1 was expressed in the transformed yeast. The GST activity of the crude extracts of yeast showed that the OsGSTL1 transgenic yeast had higher levels of GST activities than the control yeasts. These findings suggested that the OsGSTL1 was a glutathione S-transferase and could play an important role during the growth and development processes in rice.
\end{abstract}

Key words: glutathione S-transferase, GST activity, In silico, Oryza sativa L., recombinant expression, semiquantitative RT-PCR

\section{INTRODUCTION}

Glutathione S-transferases (GSTs, EC 2.5.1.18) play a significant role in the detoxification and metabolism of cellular xenobiotics and toxins by catalyzing the conjugation of these substrates with a tripeptide glutathione ( $\gamma$-glutamyl-cysteinylglycine; GSH). In the plant, the first discovered GST was from maize in 1970 and its GST activity was responsible for conjugating the chloro-Striazine atrazine with GSH (Shimabukuro et al. 1970). The GST activities, corresponding enzymes and gene sequences have been identified in the animals, plants and fungi (Wilce and Parker 1994; Sheehan et al. 2001).

The GST proteins have evolved by gene duplication to perform a range of functional roles involved in diverse intracellular events such as primary and secondary metabolisms, stress metabolism, herbicide detoxification and plant protection against xenobiotics, heavy metals and ozone damages (Lan et al. 2009; Jain et al. 2010; Mohsenzadeh et al. 2011). Classically, GSTs catalyze the transfer of the GSH to a co-substrate (R-X) containing a reactive electrophilic center to form a polar S-glutathionylated reaction product (R-SG) (Dixon et al. 2002b). Some GSTs have

*Author for correspondence: tzhu2002@yahoo.com.cn 
been shown to have glutathione peroxidase activity, with the GSTs using glutathione to reduce the organic hydroperoxides of fatty acids and nucleic acids to the corresponding monohydroxyalcohols (Cummins et al. 1999; Dixon et al. 2002a). The GSTs catalyze the isomerization of maleylacetoacetate to fumarylacetoacetate (Dixon et al. 2000). Some GSTs have ligand functions (Edwards et al. 2000) and they have been implicated as stress signaling proteins of some cell signaling pathways (Loyall et al. 2000). GSTs also have function in the cellular redox homeostasis, or regulate apoptosis (Kampranis et al. 2000; Loyall et al. 2000; Dixon et al. 2002a; Kunieda et al. 2005). The dehydroascorbic acid is reduced to ascorbic acid by DHAR in a reaction requiring glutathione (Yang et al. 2009). Microsomal-class GSTs are the members of the MAPEG (membrane-associated proteins in eicosanoid and glutathione metabolism) superfamily (Basantani and Srivastava 2007).

In this work, OsGSTL1, a member of the lambda class GST from rice, was isolated, molecularly characterized and functionally analyzed. In order to find the defined functions of this protein in the living cell, expression and activity of OsGSTL1 in yeast strain PEP4 was studied.

\section{MATERIALS AND METHODS}

\section{Plant materials and culture conditions}

The seeds of Oryza sativa $c v$. Zhonghua 11 were soaked in water for 2 days, and then grown at $28^{\circ} \mathrm{C}$ under a $16 / 8 \mathrm{~h} \mathrm{light/dark} \mathrm{photoperiod} \mathrm{at} \mathrm{an}$ intensity of approximately $250 \mu \mathrm{E} / \mathrm{m}^{2} / \mathrm{s}$. The roots and leaves of seedling stage and tillering stage, and the roots, leaves and panicles of heading stage from rice plants were collected and stored at $80^{\circ} \mathrm{C}$.

\section{Cloning and expression analysis of OsGSTL1 gene}

According to the manufacturer's protocols, total RNA was extracted from the collected rice materials using Trizol reagent (Gibco-BRL, USA). Total RNA ( $1 \mu \mathrm{g})$ was used for reverse transcriptions, which was performed to produce the first-strand cDNA with the Superscript II preamplification kit (Invitrogen, USA) and the primer Olig(dT) 18 in conditions recommended by the manufacturer. Based on the putative GST sequence (GenBank accession no. AF237487), both the primers OsGSTL1 f (5'-CACCACAAA ATGGCCGCAGCTGCAGCA) and OsGSTL1 r (5'-GGCAACCTTAAGATGCGT) were designed. A CACCACAAA sequence was introduced to the 5 '-end.of forward primer OsGSTL1 f. The OsGSTL1 sequence was amplified by reverse transcriptase-PCR using Taq DNA polymerase and 35 cycles of $94^{\circ} \mathrm{C}$ for $30 \mathrm{~s}, 58^{\circ} \mathrm{C}$ for $45 \mathrm{~s}$, and $72^{\circ} \mathrm{C}$ for $90 \mathrm{~s}$. This PCR product was cloned into pENTR/D-TOPO vector (Invitrogen, USA) and sequenced.

Semi-quantitative RT-PCR was used to detect the expression of OsGSTL1 gene in rice. A pair of primers OsGSTL1 sf (5'-CGCTTGGATTATCA GGAACT) and OsGSTL1 sr (5'-GCTTTGGA GATTAAGGGTGA) was used to produce a 300 bp amplification product. The relative expression level of OsGSTL1 in different tissues was calibrated against the expression level of the inner housekeeping gene ACTINI (McElroy et al. 1990). The primers ACTIN1 f (5'TCCGTGACATCAAGGAAAAG) and ACTIN1 $\mathrm{r}$ (5'-GATATCAACATCGCACTTCATG) were designed to get a 242 bp product. The gene fragments of OsGSTL1 and ACTIN1 were amplified using the two pairs of primers (OsGSTL1 sf/OsGSTL1 sr and ACTIN1 f/ACTIN1 r) in the PCR reaction. The amplification was carried out by the initial denaturation at $94^{\circ} \mathrm{C}$ for $2 \mathrm{~min}$, followed by 28 cycles of $94^{\circ} \mathrm{C}$ denaturation for $30 \mathrm{~s}, 58^{\circ} \mathrm{C}$ annealing for $30 \mathrm{~s}$, and $72^{\circ} \mathrm{C}$ elongation for $30 \mathrm{~s}$. The PCR products were separated in a $2.5 \%$ (W/V) agarose gel. Reactions were repeated two times for three biological replicates.

\section{In silico analysis of protein sequences}

Amino acid comparison and multiple alignments were analyzed using the ClustalW2.0 and GeneDoc software (http://www.ebi.ac.uk/Tools/msa/clustalw2/). The molecular mass, isoelectric point, grand average of hydropathy (GRAVY) and instability index were predicted using the ProtParam (http://au.expasy.org/tools/protparam.html).

Sequence identities and similarities were determined using the BLAST program with the GenBank database on the NCBI web-server (http://www.ncbi.nlm.nih.gov/Blast). Motif analysis was performed using the Pfam program (http://www.ebi.ac.uk/Tools/InterProScan/). The analysis of the signal peptide, subcellular localization and protein hydropathy was done by 
constructing the hydropathy plots with Kyte and Doolittle algorithm (http://ipsort.hgc.jp/) (Kyte and Dolittle 1982). Phylogenetic tree was constructed with the Neighbor Joining method using the MEGA4.0 program (Saitu and Nei 1987).

\section{Expression and detection of OsGSTL1 gene in yeast}

The OsGSTL1 gene was cloned into pYTV vector (Invitrogen, USA) by the LR reaction, transformed into E.coli. $\mathrm{DH} 5 \alpha$, and then the correct expression vector were transformed into yeast strain PEP4. The OsGSTL1 protein was expressed in PEP4 cell after $19 \mathrm{~h}$ incubation with SC-Ura medium, including $2 \%$ glucose and $4 \mathrm{~h}$ induction with $2 \%$ galactose. Western blot was used to detect the expression of OsGSTL1. The crude protein extracts were prepared from the yeast. The yeast was homogenized in the lysis buffer $(50 \mathrm{mM}$ Tris pH 7.5, $1 \mathrm{mM}$ EDTA, $100 \mathrm{mM} \mathrm{NaCl}, 0.1 \%$ TritonX-100, $1 \mathrm{mM}$ DTT, $1 \mathrm{mM}$ PMSF) and centrifuged at $4^{\circ} \mathrm{C}$. The supernatant was extracted with trichloroacetic acid and centrifuged at $4{ }^{\circ} \mathrm{C}$ and then the precipitate was washed two times with ethanol. Protein concentration in the crude extracts was determined with the BioRad Protein Assay Kit (Bradford 1976). The crude protein extracts $(15 \mu \mathrm{g})$ were separated in the SDS-PAGE and transferred onto Immobilon membrane (Millipore, USA) with a semi-dry transfer cell (Millipore, USA). The recombinant OsGSTL1 polypeptides expressed in the yeast strain PEP4 was detected with an antibody raised against Histag (Novagen, USA).

\section{Activity assay of OsGSTL1}

Protein concentration in the crude extracts was adjusted to $0.5 \mathrm{mg} / \mathrm{mL}$. The GST activity was measured spectrophotometrically (Habig et al. 1974; Takesawa et al. 2002). One unit of activity was defined as the amount of enzyme required forming $1 \mu \mathrm{M}$ product per minute at $30^{\circ} \mathrm{C}$.

\section{RESULTS}

\section{Expression characters of $O S G S T L 1$ in rice}

The total RNA isolated from rice plant samples was used as the template to synthesize the first strand cDNA through the reverse transcription polymerase chain reaction with primer olig(dT)18. Using the first strand cDNA as the template, semi- quantitative RT-PCR was performed with the two pairs of primers (OsGSTL1 sf-OsGSTL1 sr and ACTIN1 f-ACTIN1 r) in a PCR reaction. Both, the 300-bp OsGSTL1 fragment and 242-bp ACTINI fragment were amplified. The mRNA of OsGSTL1 was detected in the roots and leaves of rice plants at seedling and tillering stages, and their roots, leaves and panicles at the heading stage, which suggested that OsGSTL1 played an important role during the growth and development processes in the rice (Fig. 1).

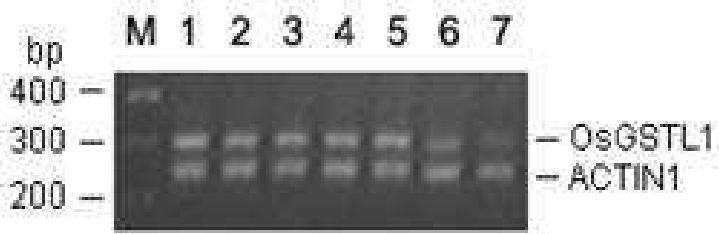

Figure 1 - The expression analysis of OsGSTL1. The expression analysis of OsGSTL1 by semiquantitative RT-PCR in rice plants. $M$ is DNA marker, lane 1 and 2 are the roots and leaves of seedling stage, lane 3 and 4 are the roots and leaves of tillering stage, lane 5, 6 and 7 are the roots, leaves and panicles of heading stage, respectively.

\section{Molecular characterization of OsGSTL1}

The sequence analysis indicated that the fulllength open reading frame (ORF) of OsGSTL1 cDNA was $732 \mathrm{bp}$ and encoded a putative polypeptide of 243 amino acids with a calculated molecular mass of $27.30 \mathrm{kDa}$ and a theoretical pI of 5.50. The predicted proteins of OsGSTL1 showed preponderance of Ala and Leu that constituted 11.5 and $10.7 \%$, respectively, but low content of Cys, Trp and Met residues that constituted $0.8 \%$ only. The alignment of the peptide sequences showed that the OsGSTL1 were a glutathione S-transferase, lambda class from Oryza sativa (OsGSTL). The significant primer structural feature for all the members of the lambda class GST have a cysteine residue in active center, which distinguishes them from other class members, which have a serine residue in same site (Dixon et al. 2002a). The OsGSTL1 protein showed a highly conserved Cys42 (Fig. 2). The motif search analysis using InterProScan revealed that the OsGSTL1 contained a N-terminal thioredoxin-fold domain (G3DSA:3.40.30.10) and a C-terminal alpha helical domain (G3DSA:1.20.1050.10) (Fig. 2). Protein blast analysis with the GenBank database on the NCBI 
web-server showed that the conserved domain "GST_C_Lambda" was in position 117 to 237. The OsGSTL1 protein was predicted to be cytoplasmic due to the absence of N-terminal signal peptides and transmembrane domains.
The hydropathy plot revealed that the OsGSTL1 protein was strongly hydrophilic with the grand average of hydropathy (GRAVY) value of -0.182 (Fig. 3). The instability index was computed to be 40.13 (Kyte and Doolittle 1982).

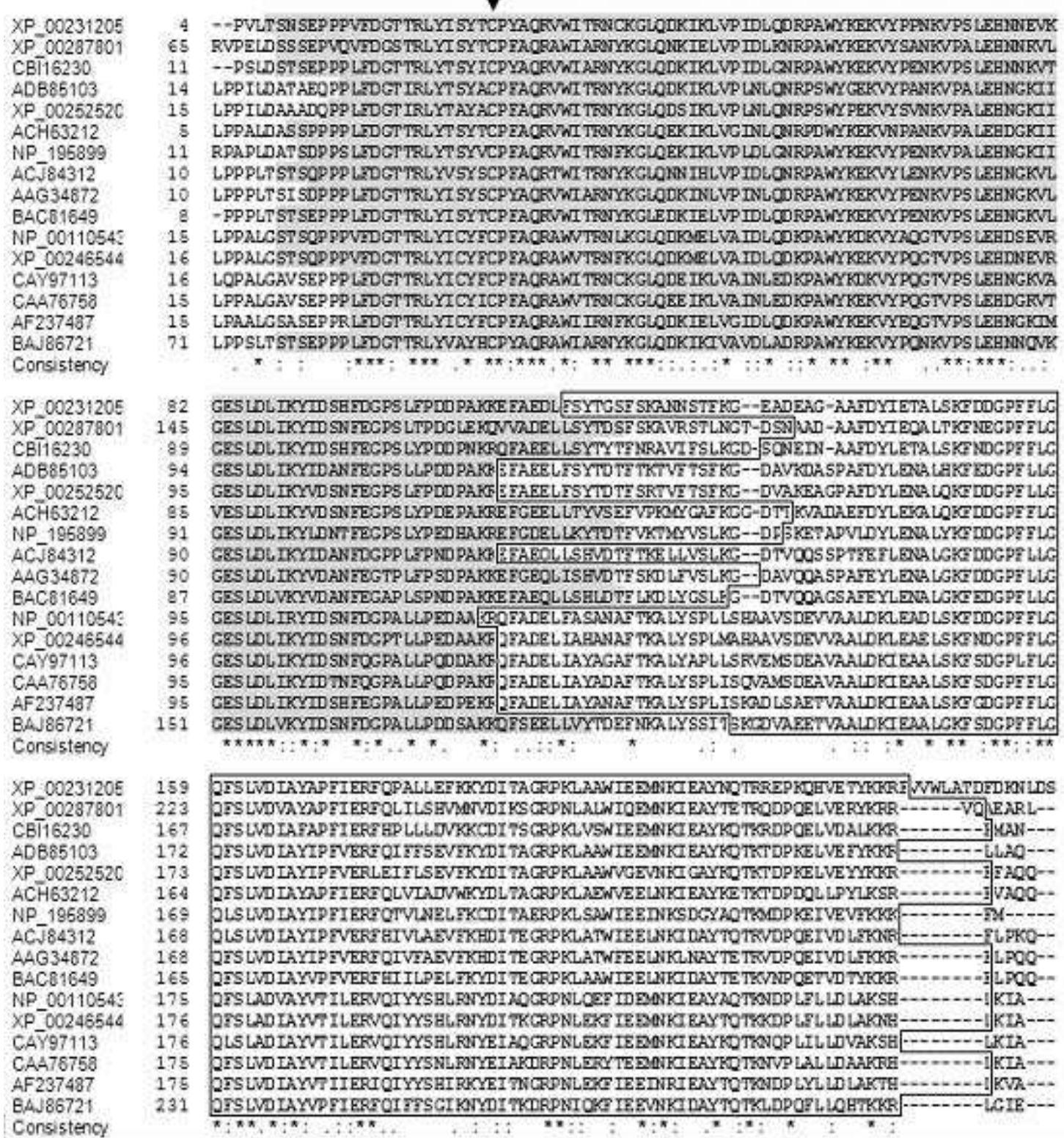

Figure 2 - Multiple sequence alignment of lambda class GSTs from various higher plants. Asterisk, identical residues; colon, highly conserved residues; dot, weakly conserved residues; dash, gaps introduced for optimal alignment. In $\mathrm{N}$-terminal region, amino acids shaded predicted to adopt $\mathrm{N}$-terminal thioredoxin-fold domains. Cterminal alpha helical domains were boxed in C-terminal region. $\boldsymbol{\Delta}$ indicates the position of the active site cystein residues. The GenBank accession numbers of proteins used for analysis from various higher plants: XP_002312053 (Populus trichocarpa, POPTRDRAFT_873617); XP_002878012 (Arabidopsis lyrata subsp. lyrata, ARALYDRAFT_906920); CBI16230 (Vitis vinifera, VIT_00025083001); ADB85103 (Jatropha curcas); XP_002525204 (Ricinus communis, RCOM_0819940); ACH63212 (Rheum australe); NP_195899 (Arabidopsis thaliana, GSTL3); ACJ84312 (Medicago truncatula); AAG34872 (Glycine max, In2-1); BAC81649 (Pisum sativum, GST); NP_001105433 (Zea may, IN2-1); XP_002465442 (Sorghum bicolor, Sb01g038900); CAY97113 (Alopecurus myosuroides, gstl1); CAA76758 (Triticum aestivum, cla30); AAF70831 (Oryza sativa, OsGSTL1); BAJ86721 (Hordeum vulgare subsp. Vulgare). 


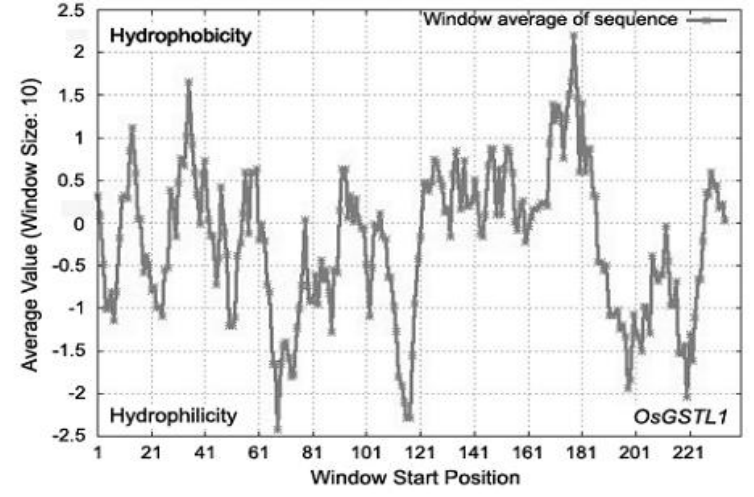

Figure 3 - Hydropathic index plot of the predicted OsGSTL1 amino acid sequence analyzed. Amino acid position is plotted on the $\mathrm{x}$ axis beginning with the N-terminus. Regions under a hydropathy score of zero are hydrophilic.

\section{Phylogenetic relationship between related OsGSTLs}

The phylogenetic relationship between the related lambda class GSTs from different plants was analyzed. A dendrogram showing the clustering of OsGSTL1 with other lambda class GSTs was constructed. The distances from the nodes, i.e., the branch lengths denoted in the tree corresponded to the sequence divergence. The lambda class GSTs was separated into two distinct subgroups, the dicots subgroup and monocots subgroup. The phylogenetic analysis revealed that the OsGSTL1 was closer to IN2-1 of Z. may, followed by Sb01g038900 of S. bicolor (Fig. 4).

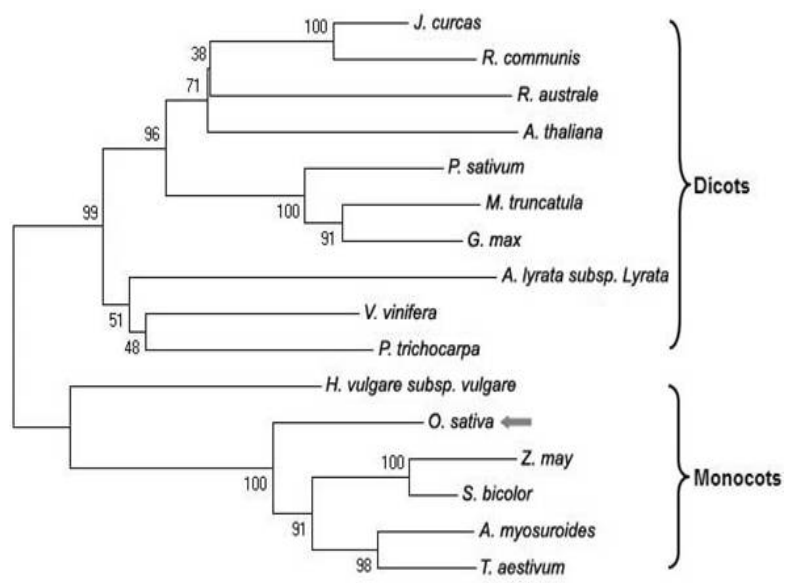

0.05

Figure 4 - Phylogenetic tree of GSTLs from plants. The lambda class GSTs was easily separated two distinct subgroups that is dicots subgroup and monocots subgroup. The OsGSTL1 of rice (arrowhead) belong to monocots subgroup. This phylogenetic tree was created using the sequences listed in Figure 2.

\section{Expressionof OsGSTL1 transgene in yeast}

The OsGSTL1 gene was cloned into pYTV vector (Invitrogen, USA) and was transformed into the yeast strain PEP4 to investigate the function of OsGSTL1. The results of Western blot showed a novel polypeptide that contained OsGSTL1 protein. The pYTV Tag fusion protein was expressed in the transformed yeast, whereas no exogenous OsGSTL1 was detected in the nontransformed yeast (Fig 5).

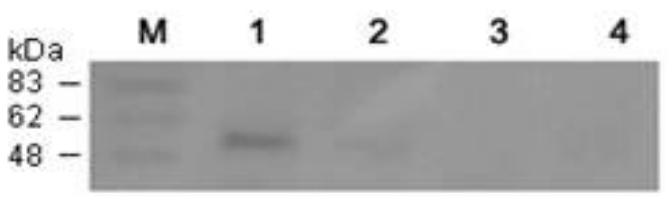

Figure 5 - Western-blot analysis of crude protein extract from OsGSTL1 transgenic yeast. M, protein marker; 1. OsGSTL1 transgenic yeast induced by galatose; 2 . untreated OsGSTL1 transgenic yeast; 3 . non-transgenic yeast induced by galatose; 4. untreated non-transgenic yeast.

\section{Determination of GST activity of recombinant OsGSTL1}

The GST activity of the crude extracts of yeast was measured with CDNB as the substrate. The data represent GST activity \pm standard deviations (SD) with three replicates. The OsGSTL1 transgenic yeast that was induced with $2 \%$ galactose, had higher levels of the GST activities than the control yeasts, which had seven more times higher GSH conjugating activity than the non-transgenic strain (Fig. 6). There was very significant difference in the GST activity between the transgenic yeast and non-transformed yeast $(p<0.001)$. The findings in this study supported the conclusion that OsGSTL1 was a member of plant glutathione S-transferase family.

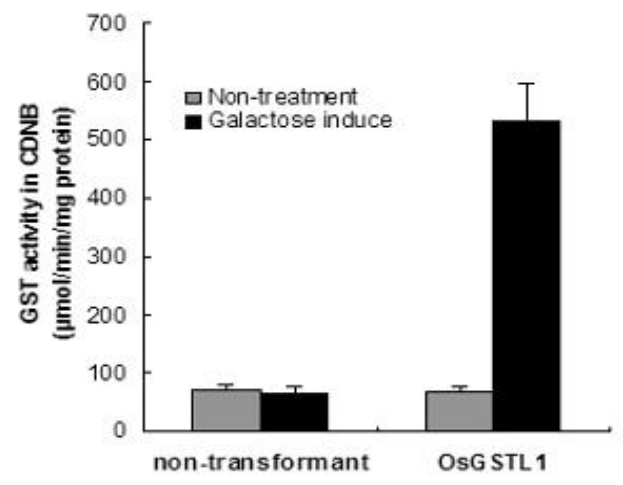

Figure 6 - Specific activities of GST in protein extracts from the OsGSTL1 transgenic yeast. The data are means of three independent experiments \pm SD. 


\section{DISCUSSION}

The plant GST gene super-families are large and highly diverse. Cloning of the plant GSTs to-date has predominantly been achieved through the large-scale mutagenesis experiments, EST and differential display approaches, and genome sequencing projects such as with Arabidopsis, rice, and maize (McGonigle et al. 2000; Alonso et al. 2003). Currently, large numbers of GST genes have been identified, or annotated from at least 17 plant species (Basantani and Srivastava 2007; Conn et al. 2008; Chronopoulou and Labrou 2009). There are 53 members in Arabidopsis, over 25 in soybean (Glysine max L.), 42 in maize (Zea mays L.), 59 in rice (Oryza sativa L.) and 81 GST genes have been genome-widely identified in Populus trichocarpa. (McGonigle et al. 2000; Dixon et al. 2002b; Wagner et al. 2002; Soranzo et al. 2004; Chi Y et al. 2011). Based on protein homology and gene organization, plants GSTs have been categorized into eight classes: phi (GSTF), zeta (GSTZ), tau (GSTU), theta (GSTT), lambda (GSTL), dehydroascorbate reductase (DHAR), tetrachlorohydroquinone dehalogenase (TCHQD) and Microsomal-class GSTs (Mohsenzadeh et al. 2011; Que et al. 2011).

In the present study, a lambda class GST subunit encoding gene, OsGSTL1, from Oryza sativa $c v$. Zhonghua 11 was cloned and characterized. The ORF of the OsGSTL1 was $732 \mathrm{bp}$, which encoded a 243 amino acids polypeptide. The protein sequences of OsGSTL1 exhibited typical feature of the lambda class GST, which contained the conserved domain "GST_C_Lambda" in Cterminal alpha helical domain and a highly conserved Cys42 in active center. The eukaryotic expression and activity assay of the OsGSTL1 showed that the OsGSTL1 protein had high activity to CDNB substrate. These results supported the conclusion that OsGSTL1 was a member of plant glutathione S-transferase family. The mRNA of OsGSTL1 was found in all the tissues of adult and young rice, which indicated that OsGSTL1 might play an important role during the growth and development processes in rice.

Some reports have shown that lambda GSTs have no GSH conjugating, for example, maize In2-1, soybean Gmin2-1, AtGSTL1 and AtGSTL2 (McGonigle et al. 2000; Dixon et al. 2002a) However, transgenic yeast strains with OsGSTL1 had seven more times higher GSH conjugating activity than non-transgenic strain. The measured values of GSH conjugating activity towards the CDNB for OsGSTL1 were evidently higher than that of OsGSTF5, AtGSTU26 and AtGSTF9 (Nutricati et al. 2006; Cho et al. 2007). But the values of GSH conjugating activity for these GSTs were obtained by the prokaryotic expression proteins, which were different from the results from eukaryotic system. The result that Arabidopsis lambda GSTs, maize IN 2-1 and soybean Gmin 2-1 lacked GSH conjugating activity also was from the prokaryotic expression system. The OsGSTL1 was also expressed in the prokaryotic expression system, but the GSH conjugating activity was not fond (data not shown). The finding demonstrated that there were some differences between the prokaryotic expression system and eukaryotic system for the lambda class GSTs. Probably the activities of GSTs might be affected by the posttranslational modification, which lacked in the prokaryotic system.

In summary, the OSGSTL1 was isolated and characterized from the rice and analyzed for its activity in the yeast strain PEP4. The protein sequences of OsGSTL1 exhibited typical feature of the lambda class GST. The eukaryotic expression and activity assay of the OsGSTL1 also demonstrated the OSGSTL1 as a member of plant glutathione S-transferase family. It is hoped that this study involving the overexpression of OsGSTLIin yeast strain PEP4 would offer the valuable information for understanding the molecular action mechanism of the lambda class GSTs and a solid basis for the development of the crops with enhanced resistance to diverse stresses.

\section{ACKNOWLEDGEMENTS}

This study was supported by the China National Transgenic Major Program (2009ZX08009-109B), the Natural Science Foundation Project of Chongqing Science and Technology Committee (CSTC, 2012jjA80009) and the Natural Science Foundation Project of Chongqing Education Committee (KJ121106, KJ131101).

\section{REFERENCES}

Alonso JM, Stepanova AN, Leisse TJ, Kim CJ, Chen H, Shinn $\mathrm{P}$ et al. Genome-wide insertional mutagenesis of Arabidopsis thaliana. Science. 2003; 301: 653-657. 
$\mathrm{Hu}, \mathrm{T}$. et al.

Bradford NM. A rapid and sensitive method for the quantitation of microgram quantities of protein utilizing the principle of protein-dye binding. Anal Biochem. 1976; 72: 248-254.

Basantani M, Srivastava A. Plant glutathione transferases: a decade falls short. Can J Bot. 2007; 85: 443-56.

Chi Y, Cheng Y, Vanitha J, Kumar N, Ramamoorthy R, Ramachandran S, Jiang SY. Expansion mechanisms and functional divergence of the glutathione s-transferase family in sorghum and other higher plants. DNA Res. 2011; 18: 1-16.

Chronopoulou EG, Labrou NE. Glutathione transferases: emerging multidisciplinary tools in red and green biotechnology. Recent Pat Biotechnol. 2009; 3: 211-223.

Cho HY, Lee HJ, Kong KH. A Phi Class Glutathione Stransferase from Oryza sativa (OsGSTF5): Molecular Cloning, Expression and Biochemical Characteristics. $J$ Biochem Mol Biol. 2007; 40: 511-516.

Conn S, Curtin C, Be'zier A, Franco C, Zhang W. Purification, molecular cloning, and characterization of glutathione S-transferases (GSTs) from pigmented Vitis vinifera L. cell suspension cultures as putative anthocyanin transport proteins. J Exp Bot. 2008; 59: 3621-3634.

Cummins I, Cole DJ, Edwards R. A role for glutathione transferases functioning as glutathione peroxidases in resistance to multiple herbicides in black-grass. Plant $J$. 1999; 18: 285-292.

Dixon DP, Cole DJ, Edwards R. Characterisation of a zeta class glutathione transferase from Arabidopsis thaliana with a putative role in tyrosine catabolism. Arch Biochem Biophys. 2000; 384: 407-412.

Dixon DP, Davis BG, Edwards R. Functional divergence in the glutathione transferase superfamily in plants. Identification of two classes with putative functions in redox homeostasis in Arabidopsis thaliana. J Biol Chem. 2002a; 277: 30859-30869.

Dixon DP, Lapthorn A, Edwards R. Plant glutathione transferases. Genome Biol. 2002b; 3: 30004.1-30004.10.

Edwards R, Dixon DP, Walbot V. Plant glutathione $S$ transferases: enzymes with multiple functions in sickness and in health. Trends Plant Sci. 2000; 5: 193198.

Habig WH, Pabst MJ, Jakoby WB. Glutathione $S$ transferase: the first enzymatic step in mercapturic acid formation. J Biol Chem. 1974; 249: 7130-7139.

Jain M, Ghanashyam C, Bhattacharjee A. Comprehensive expression analysis suggests overlapping and specific roles of rice glutathione S-transferase genes during development and stress responses. BMC Genomics. 2010; 11: 73.

Kampranis SC, Damianova R, Atallah M, Toby G, Kondi G, Tsichlis PN, Makris AM. A novel plant glutathione S-transferase/peroxidase suppresses Bax lethality in yeast. J Biol Chem. 2000; 275: 29207-29216.

Kunieda T, Fujiwara T, Amano T, Shioi Y. Molecular cloning and characterization of a senescence-induced tau-class Glutathione S-transferase from barley leaves. Plant Cell Physiol. 2005; 46: 1540-1548.

Kyte J, Dolittle RF. A simple method for displaying the hydropathic character of a protein. J Mol Biol. 1982; 157: 105-132.
Lan T, Yang ZL, Yang X, Liu YJ, Wang XR, Zeng QY. Extensive Functional Diversification of the Populus Glutathione S-Transferase Supergene Family. Plant Cell. 2009; 21: 3749-3766.

Loyall L, Uchida K, Braun S, Furuya M, Frohnmeyer H. Glutathione and a UV light-induced glutathione Stransferase are involved in signaling to chalcone synthase in cell cultures. Plant Cell. 2000; 12: 19391950.

McElroy D, Rothenberg M, Wu R. Structural characterization of a rice actin gene. Plant Mol Biol. 1990;14: 163-171.

McGonigle B, Keeler SJ, Lau SM, Koeppe MK, O'Keefe DP. A genomics approach to the comprehensive analysis of the glutathione S-transferase gene family in soybean and maize. Plant Physiol. 2000; 124: 1105-1120.

Mohsenzadeh S, Esmaeili M, Moosavi F, Shahrtash M, Saffari B, Mohabatkar H. Plant glutathione S-transferase classification, structure and evolution. Afr J Biotechnol. 2011; 10: 8160-8165.

Nutricati E, Miceli A, Blando F, De Bellis L. Characterization of two Arabidopsis thaliana glutathione S-transferases. Plant Cell Rep. 2006; 25: 997-1005.

Que Y, Liu J, Xu L, Guo J, Xu J, Chen R. Molecular cloning and expression analysis of a zeta-class glutathione S-transferase gene in sugarcane. Afr J Biotechnol. 2011; 10: 7567-7576.

Saitu N, Nei M. The neighbor-joining method: a new method for reconstructing phylogenetic tress. Mol Biol Evol. 1987; 4: 406-425.

Sheehan D, Meade G, Foley VM, Dowd CA. Enzymatic mechanism Structure, function and evolution of glutathione transferases: implications for classification of non-mammalian members of an ancient enzyme superfamily. Biochem J. 2001; 360: 1-16.

Shimabukuro RH, Swanson HR, Walsh WC. Glutathione conjugation. Atrazine detoxification mechanism in corn. Plant Physiol. 1970; 46: 103-107.

Soranzo N, Sari Gorla M, Mizzi L, Toma G, Frova C. Organization and structural evolution of the rice glutathione S-transferase gene family. Mol Genet Genomics. 2004; 271: 11-21.

Takesawa T, Ito M, Kanzaki H, Kameya N, Nakamura I. Over-expression of glutathione $S$-transferase in transgenic rice enhances germination and growth at low temperature. Mol Breed. 2002; 9: 93-101.

Wagner U, Edwards R, Dixon DP, Mauch F. Probing the diversity of the Arabidopsis glutathione $S$-transferase gene family. Plant Mol Biol. 2002; 49: 515-532.

Wilce MCJ, Parker MW. Structure and function of glutathione S-transferases. Biochim Biophys Acta. 1994; 1205: 1-18.

Yang HL, Zhao YR, Wang CL, Yang ZL, Zeng QY, Lu H. Molecular characterization of a dehydroascorbate reductase from Pinus bungeana. J Integr Plant Biol. 2009; 51: 993-1001. 